\title{
Characteristics of Deterministic and Stochastic Sandpile Models in a Rotational Sandpile Model
}

\author{
S. B. Santra, S. Ranjita Chanu and D. Deb \\ Department of Physics, \\ Indian Institute of Technology Guwahati, \\ Guwahati-781039, Assam, India.
}

(Dated: November 18, 2018)

\begin{abstract}
Rotational constraint representing a local external bias generally has non-trivial effect on the critical behavior of lattice statistical models in equilibrium critical phenomena. In order to study the effect of rotational bias in a out of equilibrium situation like self-organized criticality, a new two state "quasi-deterministic" rotational sandpile model is developed here imposing rotational constraint on the flow of sand grains. An extended set of new critical exponents are found to characterize the avalanche properties at the non-equilibrium steady state of the model. The probability distribution functions are found to obey usual finite size scaling supported by negative time autocorrelation between the toppling waves. The model exhibits characteristics of both deterministic and stochastic sandpile models.
\end{abstract}




\section{INTRODUCTION}

The phenomenon that a class of externally slow driven systems evolves naturally into a state of no single characteristic size or time without fine tuning of any parameter is known as self-organized criticality (SOC) [1, 2, 3]. In SOC, the system evolves into a non-equilibrium steady state characterized by long range spatio-temporal correlations and power law scaling behavior as observed in equilibrium critical phenomena[4]. The phenomenon is observed in many natural physical and chemical processes 5]. Sandpile, a prototypical model, was introduced by Bak, Tang and Wiesenfeld (BTW) [6] for studying SOC. The system here is driven by adding sand grains, one at a time, randomly to the sites of a regular lattice. In BTW, the model evolves following certain deterministic rules for distributing grains of a sand column among all the nearest neighbors equally if the height of a column attains a predefined critical height. This intermittent burst of toppling activity is called avalanche. Soon after the introduction of BTW sandpile model, a stochastic version of the model namely Manna's stochastic model (MSM)[7] was proposed. In MSM, sand grains flow in two randomly selected directions out of four possible directions on a square lattice after toppling. It was initially believed that BTW and MSM belong to the same universality class [8, 9, 10]. However, calculating an extended set of exponents, introduced by Christensen and Olami[11], Ben-Hur and Biham claimed for the first time that MSM belongs to a new universality class[12]. Different universality class of MSM has been confirmed later by performing multifractal[13] and moment[14] analysis of the avalanche distribution functions. It is found that the distribution functions of avalanche properties obey finite size scaling (FSS) in MSM[10, 15] whereas in BTW, some of them obey multi-scaling[13, 14].

External bias fields were found to have non-trivial effect on the critical properties of various lattice statistical models in equilibrium critical phenomena. For example, in directed self-avoiding walks (DSAW)[16], spiral self-avoiding walks (SSAW)[17], spiral lattice animal (SLA) [18], directed percolation (DP) [19], spiral percolation (SP) [20], directed spiral percolation (DSP) [21], etc, the external bias has changed the critical behavior of these models. The effect of external bias on SOC is studied so far applying external global directional bias only. There are two such models, directed sandpile model (DSM) introduced by Dhar and Ramaswamy[22] and directed fixed energy sandpile (DFES) model introduced by Karmakar and Manna [23]. In these models, sand grains flow in a globally preferred direction after top- 
pling. Application of global bias on the sandpile model introduces anisotropy in the systems and leads to new universality classes for DSM[22] and DFES[23]. In contrary, the lattice statistical models like SLA and SP not only remain isotropic under a local rotational bias but also exhibit nontrivial critical behavior [18, 20]. It is then intriguing to study the effect of a rotational constraint on the critical properties of sandpile models at out of equilibrium situation.

In this paper, a new two state "quasi-deterministic" rotational sandpile model (RSM) is introduced imposing rotational constraint on the flow of sand grains. In this model, a site topples if it exceeds a predefined critical height and two sand grains flow, one in the forward direction, the direction from which the last sand grain was received and the other in a specific rotational direction, say clockwise with respect to the forward direction. Since the direction of last sand grain received varies site to site, the rotational constraint is local in nature. During avalanche, the direction of the last grain received may change if the toppling sequence is changed. This introduces certain "randomness" or "internal stochasticity" in the model. RSM includes features like mass conservation, open boundary, local deterministic rule for grain distribution along with toppling imbalance, certain stochasticity. The model is studied here numerically in two dimensions $(2 D)$ and the critical avalanche properties at the non-equilibrium steady state are characterized. An extended set of critical exponents are calculated, nature of the scaling functions are determined, and time auto correlations between the toppling waves are analyzed. Interestingly, it is found that some of the critical exponents are similar to that of BTW but different from that of MSM whereas the scaling functions follow usual FSS as in MSM. Consequently RSM belongs to a new universality class. A physical realization of RSM could be a sandpile on a disk rotating slowly about an axis perpendicular to the plane of the disk and passing through the center of the disk.

\section{ROTATIONAL SANDPILE MODEL}

RSM is defined here on a square lattice of size $L \times L$ in two dimensions $(2 D)$. A positive integer $h_{i}$, the height of the sand column, is assigned to each lattice site. Initially, all $h_{i} \mathrm{~s}$ are set to zero. Sand grains are added, one at a time, to randomly chosen lattice sites and the variable $h_{i}$ is incremented to $h_{i}+1$ if a sand grain is added to the $i$ th site. A site is called active or critical when the height of a site becomes greater than or equal to 
a predefined threshold value $h_{c}=2$, as in MSM. The active site will burst into a toppling activity. Toppling of the first active site initiates an avalanche. This site can be called the origin of the avalanche. The flow of sand grains is now demonstrated with the help of Fig, 1, On the very first toppling of the active site, the central black circle, two sand grains are given away to two randomly selected nearest neighbors out of four nearest neighbors on a square lattice. Note that this is the only externally imposed stochastic step in the model. The grey circles (sites 2 and 4) represent the recipient sites. As soon as a site receives a sand grain, the direction $d_{i}$ from which the grain was received is assigned to it along with the increment of its height $h_{i}$. The value of $d_{i}$ can change from 1 to 4 as there are four possible directions on a square lattice. The solid arrows in Fig 1 indicates different possible directions. As the avalanche propagates, the direction $d_{i}$ and height $h_{i}$ are updated on receiving a sand grain and the information from which direction the last sand grain was received is only kept. Now the next active sites with $h_{i} \geq 2$ in the avalanche will topple following a deterministic rule. Two sand grains from a critical site will flow, one in the forward direction, the direction from which the last grain was received, and the other in a clockwise rotational direction with respect to the forward direction. Note that, distribution of sand grains from an active site depends on the receiving direction of the last sand grain before toppling which varies site to site. Thus, the rotational constraint represents a spatially local bias here in RSM in contrast to the directed bias where the directions of sand flow are globally fixed. If the grey sites (2 and 4) in Fig 1 are the new active sites after receiving sand grains from the central site, the flow of sands after toppling of the grey sites will be in the forward and along a rotational direction as indicated by dotted arrows. The toppling rules for the $i$ th active site can be stated as

$$
\begin{aligned}
h_{i} & \rightarrow h_{i}-2 \\
h_{j} & \rightarrow h_{j}+1, j=d_{i} \& d_{i}+1
\end{aligned}
$$

where $d_{i}$ is the direction from which the last sand grain was received by the $i$ th site. If the index $j$ becomes greater than 4 it is taken to be 1 . It is important to note that the number of sand grains outgoing from a site after toppling is not necessarily equal to the number of sand grains incoming to the same site after toppling of its nearest neighbors once each. Therefore, there is toppling imbalance in RSM as in MSM[24] whereas there is complete toppling balance in BTW. Note that, toppling imbalance in RSM is due to the imposition of rotational constraint whereas the same appears in MSM due to stochasticity in 
grain distribution. Toppling of an active site may cause a series of intermittent bursts which constitutes an avalanche. During an avalanche no sand grain is added. Propagation of an avalanche stops if all sites of the lattice become under critical. The avalanche dynamics is studied with open boundary condition. The number of sand grains remains conserved in the model. The steady state corresponds to the constant average height of the sandpile when the current of incoming flux of sand grains is equal to that of the outgoing flux.

It should be noted here that the final state in an avalanche depends on the sequence of toppling of the critical sites due to the rotational rules considered here. It is demonstrated in Fig 2 considering two nearest neighbors at the critical state $(h=2)$ at the same time step. The numbers represent the height of the sand column at that site. The short arrows associated with the numbers represent the direction from which the last sand grain was received. It can be seen that reversing the order of toppling, two different final states are obtained. It has been verified for a larger lattice that the interchange of toppling sequence leads to different final configurations starting from the same initial state. Consequently, the model is non-abelian. Note that toppling of one site may change the present state (direction from which the last sand grain was received) of the nearest neighbors. Consequently, the sand grains will flow in different directions than it was expected before toppling of its neighbors. Apart from the initial stochastic step, the imposition of the rotational rule then also introduces some randomness in the model during time evolution. This can be considered as "internal stochasticity" in the model in contrast to the externally imposed stochasticity in MSM. This inherent randomness makes the model not only non-abelian but also "quasideterministic". Due to the stochastic dynamical rules, MSM had already been found nonabelian[25]. It is also important to notice that the local correlation in the rotational toppling rule then can not propagate throughout the avalanche as in BTW because of the "internal stochasticity" in the model.

RSM is thus a new two state, "quasi-deterministic", non-abelian sandpile model under local external rotational bias. Despite few attempts, the critical behavior of non-abelian sandpile models are less understood. The non-abelian Zhang model [26] belongs to the same universality class as that of abelian BTW in the limit of small quantum of energy added to the system[27]. Directed slope model is non-abelian[28] but it shows the same critical behavior as that of the abelian directed model[22]. A crossover behavior of critical exponents from a generalized Zhang model to that of BTW model depending upon a non universal parameter 
$p$, the probability of sand flow in a given direction, is shown by Biham et al[29]. On one hand, RSM has features of mass conservation, open boundary, local deterministicity in gain distribution to the neighborhood after toppling as that of BTW, on the other hand, it has features like toppling imbalance, certain stochasticity and non-abelianity as that of MSM. It is then interesting to characterize the critical properties of RSM which has microscopic features of both BTW and MSM. Below, the avalanche properties at the steady state are characterized in three different ways: $(i)$ calculating an extended set of critical exponents, (ii) performing moment analysis of the probability distribution functions and (iii) coarsening an avalanche into a series of toppling waves.

\section{RESULTS AND DISCUSSION}

\section{A. The steady state}

The non-equilibrium steady state is defined by the constant average height of the sandpile at which the current of influx of sand grain to the system is equal to the current of out flux of the same at the open boundary. In order to identify the steady state, the average height

$$
\langle h\rangle=\frac{1}{L^{2}} \sum_{i=1}^{L^{2}} h_{i}
$$

has been measured generating a large number of avalanches. The average height $\langle h\rangle$ is plotted against the number of avalanches upto $10^{6}$ in Fig 3 for the system size $L=2048$. It can be seen that a constant average height $\langle h\rangle$ is achieved and it remains constant over a large number of avalanches. For smaller lattice sizes the steady states are reached by smaller number of avalanches. A slight variation of the average height with the system size is observed. The values of $\langle h\rangle$ against the system size $L$ is shown in the inset. In order to

characterize the physical properties of the avalanches occurred at the non-equilibrium steady state, simulations have been performed on the square lattice of sizes $L=128$ to $L=2048$ in multiple of 2 . First $10^{6}$ avalanches were skipped to achieve the steady state. Extensive data collection have been made for each lattice size for averaging, ranging from $32 \times 10^{6}$ avalanches for $L=128$ down to $2 \times 10^{6}$ avalanches for $L=2048$ in ten configurations. In each configuration, the initial $10^{5}$ avalanches are neglected again on the steady state before collecting data. It should be mentioned here that due to the rotational constraint 
the lifetime of an avalanche in RSM is much higher in comparison to that in other models. Generation of large number of avalanche then requires huge computer time in RSM.

\section{B. Avalanche cluster Morphology}

A comparison of the morphology of avalanche clusters in the steady state of different sandpile models is made here. Typical large avalanche clusters of BTW, MSM and RSM obtained in their respective steady states are shown in Fig 4 (color online). The avalanche clusters are generated on a square lattice of size $64 \times 64$ dropping sand grains one at a time at the center of the lattice. The clusters shown here have 21 maximum number of toppling in each and it is represented by the red color. Different colors correspond to different number of toppling of sites in an avalanche as: red for 21, green for 20-17, blue for 16-13, orange for 12-9, magenta for 8-5, and grey for 4-1 toppling numbers. White spaces inside the avalanche correspond to the sites that did not topple at all during the avalanche. There are few things to notice. First, RSM avalanche cluster is different from that of both BTW as well as MSM. BTW avalanche cluster consists of concentric zones of lower and lower number of toppling around a single maximal toppling zone [30] whereas avalanche cluster of MSM is random in nature [12]. RSM avalanche cluster neither fully consists of concentric zones as in BTW nor it is totally random. It is important to note that the local correlation in rotational constraint does not lead to long range correlation generating BTW like correlated structure. Second, there are more than one red zones (maximum number of toppling zones) in the avalanche cluster of RSM in Fig $4(c)$ as in the avalanche cluster of MSM (Fig $4(b)$ ) whereas there is only one red zone at the center of the avalanche cluster of BTW (Fig. $4(a)$ ). It is then possible to have several maximal toppling zones in an avalanche in RSM whereas in BTW always one maximal toppling zone appears[30]. RSM avalanche looks like superimposition of several BTW like structures around different maximal toppling zones. Occurrence of several maximal toppling zones is also a common feature in MSM avalanche[12, 30]. Third, though the sand grains are added at the central site of the lattice the maximal toppling zones appear at arbitrarily different places in RSM as well as in MSM. In BTW, the central zone correspond to the maximal toppling zone $[30]$. Fourth, BTW avalanche clusters are compact without holes or no toppling regions inside an avalanche (see Fig.4 $4(a)$ ). On the other hand, there are several holes appear in MSM as seen in Fig $4(b)$. RSM avalanche cluster is almost 
compact with few holes here and there. The appearance of holes in MSM is due to the stochastic rule of sand distribution and the same in RSM is due the rotational rule of sand distribution. Note that holes of a single site also appear in the avalanche clusters of RSM. Apparently it seems generation of single sited hole is forbidden by the rotational rule. Single sited holes only could appear at the termination point of two different branching of toppling of a previous site. An avalanche can be considered as a branching process since toppling of a site can make more than one neighbors critical[31]. The RSM avalanche cluster therefore has properties of both the deterministic BTW and stochastic MSM. It is then expected that the critical avalanche properties in the steady state will show a mixed behavior. Note that, the avalanche clusters are isotropic in space in RSM as in both BTW and MSM. However, the avalanche clusters are anisotropic in the cases of DSM and DFES where two correlation lengths are required to characterize their spatial extensions.

\section{Criticality and Power laws}

In order to characterize different physical properties of the avalanches occurred at the steady state, different quantities like toppling size $s$ of an avalanche, avalanche area $a$, lifetime $t$ and spatial extension $l$ are measured. Toppling size $s$ is defined as the total number of toppling occurred in an avalanche. Avalanche area $a$ is equal to the number of distinct sites toppled in an avalanche. Lifetime $t$ of an avalanche is taken as the number of parallel updates to make all the sites under critical. Spatial extension $l$ of an avalanche is given by $l^{2}=2 \sum_{i=1}^{a}\left(\mathbf{r}_{0}-\mathbf{r}_{i}\right)^{2} / a$ where $\mathbf{r}_{0}=\sum_{i=1}^{a} \mathbf{r}_{i} / a, \mathbf{r}_{i}$ is the position vector of the distinct sites toppled. The related critical exponents are estimated determining the probability distributions of all these properties $(s, a, t$ and $l)$. The probability distribution function of an avalanche related quantity $x$ at the steady state of a given system size $L$ is expected to obey power law behavior given by

$$
P(x, L) \sim x^{-\tau_{x}} \mathbf{f}\left(x / L^{D_{x}}\right)
$$

where $\tau_{x}$ is the corresponding critical exponent and $x$ stands for $s, a, t$ and $l . \mathrm{f}\left(x / L^{D_{x}}\right)$ is the finite size scaling function and $D_{x}$ is called a capacity dimension. In the $L \rightarrow \infty$ limit, the scaling function $\mathrm{f}(0)$ become a constant and the power law behavior given in Eq 3 can be approximated as $P(x) \sim x^{-\tau_{x}}$. The corresponding exponents $\tau_{x}$ can be estimated from 
the slope of the best fitted straight line through the data points in log-log scale. Data are collected in bins of interval of 10s, 100s, 1000s and so on. Finally the data are normalized by the bin widths. In Fig 5 , the probability distribution $P(s)$ of the toppling size $s$ is plotted for different system sizes, $L=128$ to $L=2048$ in multiple of 2 . It can be seen that the toppling size distribution has the same power law behavior for different system size $L$ with a cutoff that increases with $L$. Since there is no typical toppling sizes of an avalanche at the non-equilibrium steady state, RSM then exhibits self-organized criticality. The solid line represents the best fitted straight line between the data points of $L=2048$ with a slope of $1.224 \pm 0.005$. The error is due to the least square fitting taking into account of statistical error of each data point. The slopes obtained from the best fitted part of data for other values of $L$ remain within this error bar. In order to extract the critical exponents related to other avalanche properties the same procedure has been followed. However, in Fig [6 the probability distribution $P(x)$ is plotted against $x$ only for $L=2048$ where $x$ corresponds to area $a$ (circles), lifetime $t$ (triangles) and spatial extension $l$ (squares). It can be seen that the probability distributions $P(x)$ follow reasonable power law behavior for each property $x$. The solid lines represent the best fitted straight line through the data points. The values of the associated critical exponents $\tau_{x}$ are obtained from the slopes of the best fitted straight lines as $\tau_{a}=1.334 \pm 0.005, \tau_{t}=1.389 \pm 0.005$ and $\tau_{l}=1.667 \pm 0.007$. The error bars quoted here are the least square fit errors taking into account of statistical error of each data point. A comparison of the values of the exponents obtained here is made with that of BTW and MSM in Table【. The values of the exponents for BTW and MSM are taken from Ref.[12, 32]. Interestingly, the toppling size exponent $\tau_{s}$ and the lifetime exponent $\tau_{t}$ are different whereas $\tau_{a}$ and $\tau_{l}$ are almost the same as that of BTW. The disagreement of the lifetime and toppling size distribution exponents with the corresponding BTW exponents can be accounted by the fact that in RSM the avalanche waves generally have a spiraling nature around several maximal toppling zones within the avalanche cluster and as a consequence it will take longer time and large number of toppling for an avalanche to die away than that in BTW where a single maximal toppling zone occurs and the toppling wave propagates outwardly. On the other hand, in comparison to MSM, most of the exponents are found different. Thus, from the point of view of power law correlations, some of the avalanche properties are similar to that of BTW but different from MSM. Note that $\tau_{s}=2-1 / \tau_{a}$, conjectured by Majumder and Dhar[33], is satisfied in case of MSM but it is not valid for 
BTW. It can be seen that the conjecture is just outside the error bar here in case of RSM. The expected value of $\tau_{s}$ in RSM from the conjecture is $\approx 1.25$ close to the obtained value $1.224 \pm 0.005$.

Since the avalanche properties are related to each other, conditional expectation values are defined as introduced by Christensen et al[34]. The conditional expectation value of an avalanche property $x$ when another property is exactly equal to $y$ is defined as

$$
\langle x(y)\rangle=\sum_{x} x P(x, y)
$$

where $P(x, y)$ is the probability to find a property $x$ when the other property is exactly equal to $y$ for a given system size $L$. In the steady state, the expectation values scale with its argument as

$$
\langle x(y)\rangle \sim y^{\gamma_{x y}}
$$

where $\gamma_{x y}$ is a critical exponent. Four expectation values $\langle s(a)\rangle \sim a^{\gamma_{s a}},\langle a(t)\rangle \sim t^{\gamma_{a t}}$, $\langle a(l)\rangle \sim l^{\gamma_{a l}}$, and $\langle t(l)\rangle \sim l^{\gamma_{t l}}$ are calculated on a square lattice of size $L=2048$ and their scaling behavior are determined. In Fig,7, the expectation values are plotted against their arguments in order to evaluate the exponents $\gamma_{x y}$. From the slope of the best fitted straight lines, the values of the exponents are estimated as: $\gamma_{s a}=1.453 \pm 0.003, \gamma_{a t}=1.167 \pm 0.005$, $\gamma_{a l}=2.002 \pm 0.002$ and $\gamma_{t l}=1.715 \pm 0.005$. The values of $\gamma_{x y}$ in DSM[12] are smaller in comparison to the values obtained here. In Table 【, the values of $\gamma_{x y}$ are compared with that of BTW and MSM. There are few things to notice. First, $\gamma_{s a}$ is found greater than one and a relevant exponent. This is expected because in this model, a site topples many times in an avalanche due to rotational constraint. Second, the exponent $\gamma_{a l}$ is found $\approx 2$ since the avalanche clusters are almost compact with a few holes here and there. Third, the value of the dynamical exponent $\gamma_{t l}$ is the highest in RSM and it is lowest in BTW. Because, due to the rotational constraint the sand grains rotates around several maximal zones inside the avalanche and take longer time to complete an avalanche. Fourth, according to the scaling function form given in Eq 5 , the exponents should satisfy the scaling relation $\gamma_{x z}=\gamma_{x y} \gamma_{y z}$. It can be seen that the scaling relation $\gamma_{a l}=\gamma_{a t} \gamma_{t l}$ is satisfied within error bars. Fifth, the values of $\gamma_{s a}, \gamma_{a t}$ and $\gamma_{t l}$ are found different from that of BTW as well as MSM except $\gamma_{a l}$. Finally, a set of scaling relations between the probability distribution exponents $\tau_{x}$ and the exponents $\gamma_{x y}$ describing the conditional expectation values of the avalanche properties can 
be obtained from the following identity

$$
\int\langle x(y)\rangle P(y) d y=\int\langle x(z)\rangle P(z) d z
$$

which would be satisfied by any set of three stochastic variables $x, y$ and $z$. Using this identity and the relation $\gamma_{x z}=\gamma_{x y} \gamma_{y z}$, the following scaling relation can be obtained

$$
\gamma_{x y}=\left(\tau_{y}-1\right) /\left(\tau_{x}-1\right)
$$

The above scaling relation is satisfied within error bars for $x, y \in\{s, a, l, t\}$. Thus, the extended set of exponents obtained here in RSM from both power law analysis and conditional probabilities are consistent with the scaling relations. Note that the values of the critical exponents obtained here should remain invariant under the reversal of rotational symmetry. Though some of the exponents are close to that of BTW, RSM belongs to a new universality class because the extended set of exponents are not identical. Note that, DSP and DEFS already belongs to different universality classes because of their anisotropic character. Directed models show a continuous phase transition from an absorbed phase to an active phase[22, 23, 35].

\section{Moment Analysis and Finite Size Scaling}

Now the avalanche properties are analyzed to understand the nature of the scaling functions, FSS or multi-scaling, following the method of moment analysis[13, 14, 24]. The probability distribution $P(x, L)$ of an avalanche property $x$ in a finite system of size $L$ is expected to obey a scaling function form as given in Eq. 3 , $P(x, L) \sim x^{-\tau_{x}} \mathbf{f}\left(x / L^{D_{x}}\right)$ where $D_{x}$ is called a capacity dimension as already mentioned. The finite system size $L$ causes a cutoff of the probability distributions at $x_{\max } \sim L^{D_{x}}$. The $q$-moments of a property $x$ is defined as

$$
\left\langle x^{q}\right\rangle=\int_{0}^{x_{\max }} x^{q} P(x, L) d x \sim L^{\sigma_{x}(q)}
$$

where $\sigma_{x}(q)=\left(q+1-\tau_{x}\right) D_{x}$. Thus, if the probability distributions obey FSS then the moment exponent $\sigma_{x}(q)$ should have a constant gap between two successive values, $\sigma_{x}(q+$ 1) $-\sigma_{x}(q)=D_{x}$. On the other hand, if they obey multi-scaling, $\sigma_{x}(q)$ should have a continuous dependence on $q$. For a given $q$, the value of $\sigma_{x}$ has been obtained from the slope of the plot of $\log \left\langle x^{q}(L)\right\rangle$ versus $\log L$ changing lattice size from $L=128$ to 2048 in 
multiple of 2. The least square fit error for each $\sigma_{x}$ value is found \pm 0.01 . A sequence of exponents $\sigma_{x}(q), x \in\{s, a, t\}$, is obtained for 400 values of $q$ between 0 and 4 . In Fig, 8 , the exponent related to the average toppling size $\sigma_{s}(q)$ is plotted against the moment $q$. An important point to note here is that the value of $\sigma_{s}(1)$ is $\approx 2$ in all three models. The average toppling size varies with the system size as $\langle s\rangle \sim L^{\sigma_{s}(1)}$ where $s$ is equivalent to the number of steps by a random walker required to reach the lattice boundary[31]. Thus, the value of $\sigma_{s}(1) \approx 2$ represents the diffusive character of the model, one of the characterizing features of the sandpile models. The diffusive behavior of RSM is also consistent with the fact that spiral random walks are diffusive[36]. In order to compare the results of RSM with that of BTW and MSM, a sequence of exponents $\sigma_{x}(q)$ are also obtained for these two models and plotted in the same figure. It can be seen that the variation of $\sigma_{s}(q)$ with $q$ in Fig 8 is not identical with either BTW or MSM. Further analysis of this sequence of exponents is then needed in order to understand the nature of the scaling function. The slopes $\partial \sigma_{x} / \partial q$ are then estimated using finite difference method. If the probability distributions obey multiscaling, the rate of change of $\sigma_{x}(q)$ with $q$ should not be a constant. In Fig.9, $\partial \sigma_{x} / \partial q$ s are plotted against the moment $q$ for $x \in\{s, a, t\}$ and compared with that of BTW and MSM. In Fig.9, the solid line represents the data of RSM, the dashed line represents the data of MSM and the dotted line represents the data of BTW. The rate of change of $\sigma_{x}(q)$ with respect to $q$ for all three properties $x \in\{s, a, t\}$ in RSM are different from that of both BTW and MSM and remain unchanged in higher moments as in the case of MSM. In BTW, the derivatives corresponding to toppling size $s$ and lifetime $t$ do not saturate with the moment $q$. A comparison of variation of $\partial \sigma_{x} / \partial q$ with $q$ in BTW and RSM with respect to MSM can be made. In order to have a comparative study a quantity $\Delta_{x, m}(q)$ is defined as

$$
\Delta_{x, m}(q)=\left|1-\frac{\sigma_{x, m}^{\prime}(q)}{\sigma_{x, M S M}^{\prime}(q)}\right|
$$

where $\sigma^{\prime}(q)=\partial \sigma / \partial q$ and $m$ stands for the models BTW or RSM. $\Delta_{x, m}(q)$ is plotted against $q$ in the inset of corresponding plots. The dotted line corresponds to $\Delta_{x, B T W}$ and the solid line corresponds to $\Delta_{x, R S M}$. It can be seen that the value of $\Delta_{x, R S M}(q)$ remains constant with respect to MSM whereas in case of BTW, data corresponding to toppling size and lifetime increases slowly with respect to MSM. Thus, the scaling functions of the avalanche properties in RSM follow FSS as in MSM rather than multi-scaling as in BTW.

The values of the capacity dimensions $D_{x}$ can be calculated taking the large $q$ limit 
of $\partial \sigma_{x}(q) / \partial q$. The values obtained are: $D_{s}=2.86 \pm 0.01, D_{a}=2.03 \pm 0.01$ and $D_{t}=$ $1.60 \pm 0.01$. The errors are due to the finite difference method adopted for differentiation of the $\sigma(q)$ sequence. The value of $D_{l}$ is trivially equal to 1 because $l_{\max } \sim L$. Since $\int P(x) d x=\int P(y) d y$ for a given system size $L$, it can be shown that $D_{x} / D_{y}=\gamma_{x y}$. Taking $D_{l}=1$, one should have $D_{x}=\gamma_{x l}$. For RSM, the values of $D_{x}$ and $\gamma_{x l}$ are found close. Since $\partial \sigma_{s}(q) / \partial q$ and $\partial \sigma_{a}(q) / \partial q$ do not saturate in BTW, the corresponding capacity dimensions are not possible to estimate. However, the capacity dimension $D_{a}$ in all three models are found $\approx 2$ as it is expected. The values of $D_{s}$ and $D_{t}$ in MSM (as in Ref.[10]) and RSM differ slightly. Note that $D_{s}\left(2-\tau_{s}\right) \approx 2.22$ is slightly higher than $\sigma_{s}(1) \approx 2$ because $q=1$ remains in the nonlinear regime in RSM.

Knowing the values of capacity dimensions, it is now possible to check the scaling function form given in Eq 3 studying the distribution functions for different system sizes $L$. The scaling function form is checked by plotting a scaled distribution $P(x) L^{D_{x} \tau_{x}}$ against the scaled variable $x / L^{D_{x}}$ for $x \in\{s, t\}$ in Fig 10 following Chessa et al[10]. For both the properties, toppling size $s$ and lifetime $t$, a reasonable collapse of data are observed for $L=512,1024$ and 2048 in support of the assumed scaling function form in Eq[3. In the inset of Fig 10, data collapse for toppling size $s$ is also shown in log-normal scale. The FSS forms assumed here in RSM for the avalanche properties are then correct. In spite of the fact that RSM has locally deterministic rule for grain distribution, it is conservative, its avalanche cluster morphology is almost compact, it is diffusive, and some of the critical exponents are similar to that of BTW, it is interesting to note that the scaling functions in RSM do not follow multi-scaling as in BTW. A sandpile model with microscopic as well as macroscopic characteristics of a deterministic model like BTW follows FSS which is characteristics of a stochastic model like MSM is a new result. This has happened due to the nature of the rotational constraint which incorporates "internal stochasticity" by changing the state of the critical sites in a time step during time evolution of the system. Note that, due to the presence of rotational constraint on the sand flow the toppling balance of BTW is also broken as already mentioned. Recently, it is demonstrated by Karmakar et al[24] that the scaling functions obey FSS rather than multi-scaling if toppling imbalance is introduced in the BTW sandpile model. Existence of FSS in RSM is possibly due to toppling imbalance as well as "internal stochasticity" in the model. 


\section{E. Time Autocorrelation}

FSS of avalanche properties can be confirmed by studying time autocorrelation between toppling waves. The time evolution of toppling dynamics is studied here coarsening the avalanches into a series of toppling waves [37]. Toppling waves are defined as the number of toppling during the propagation of an avalanche starting from a critical site $O$ without toppling $O$ more than once. Each toppling of $O$ creates a new toppling wave. Thus, the total number of toppling $s$ in an avalanche can be considered as

$$
s=\sum_{k=1}^{m} s_{k}
$$

where $s_{k}$ is the number of toppling in the $k$ th wave and $m$ is the number of toppling waves during the avalanche. It is then possible to generate a wave time series $\left\{s_{k}\right\}$. Following Menech and Stella[38], for a given lattice size $L$, a time autocorrelation function is defined as

$$
C(t)=\frac{\left\langle s_{k+t} s_{k}\right\rangle-\left\langle s_{k}\right\rangle^{2}}{\left\langle s_{k}^{2}\right\rangle-\left\langle s_{k}\right\rangle^{2}}
$$

where $t=1,2, \cdots$ and $\langle\cdots\rangle$ represents the time average. It has already been demonstrated by Menech and Stella [38] and Karmakar et al[24] that $C(t)$ is long range for BTW whereas it remains negative initially and then becomes zero in case of MSM. Thus, the waves in BTW have correlation over a longer period of time whereas they are uncorrelated in case of MSM. This is also argued by Menech and Stella[38] that this observation is consistent with the fact that the toppling sizs follow multi-scaling in BTW and FSS in MSM. The time autocorrelation function $C(t)$ for the toppling waves has also been calculated here in RSM for a system size $L=1024$ taking time average over $10^{6}$ toppling waves. $C(t)$ is plotted against $t$ for RSM in Fig 11. In order to compare the data of RSM with that of BTW, and MSM, $C(t) \mathrm{s}$ of these models are also calculated and plotted in the same figure. It can be seen that there is long range correlation for BTW and anti-correlation for MSM as expected. The toppling waves are also uncorrelated here in RSM as in the case of MSM. $C(t)$ is found negative initially and then becomes zero. It is then consistent with the fact that the toppling size distribution follow FSS rather than multi-scaling. The origin of negative autocorrelation in MSM is the stochasticity. In RMS, the local deterministic toppling rule

picks up certain randomness during the evolution and as a consequence the toppling wave shows negative auto correlation. Though the rotational constrain has local correlation, the 
toppling waves become uncorrelated because of the "hidden stochasticity". It should be mentioned here that in the cases of MSM and RSM, the sites involved in a toppling wave may topple more than once unlike in the case of BTW. Moreover, the toppling numbers as well as the final configurations of MSM and RSM strongly depend on the sequence of toppling. As a consequence, Eq10 may not satisfy always in RSM and MSM. However, on an average the collection of toppling waves can be considered as a representation of an avalanche in these models.

\section{CONCLUSION}

A new two state "quasi-deterministic" sandpile model, RSM, is defined imposing rotational constraint on the sand flow in order to study the effect of local external bias on self-organized critical systems. The model has microscopic properties like mass conservation, open boundary, local deterministicity in sand grain distribution on toppling as that of BTW. At the same time, the rotational bias introduces toppling imbalance and certain stochasticity at the microscopic level as in MSM. The non-equilibrium steady state of RSM is characterized by power law distribution of avalanche properties. The avalanche cluster morphology in the steady state exhibits characteristics of both BTW as well as MSM. RSM is found to be diffusive in character like other sandpile models. Calculating an extended set of critical exponents it is found that some of the exponents are close to that of BTW but different from MSM. The values of the exponents satisfy the scaling relations among them within error bars. RSM then belongs to a new universality class. The scaling function forms are determined. It is found that the scaling functions obey usual FSS as in the case of MSM rather than multi-scaling as in the case of BTW. This has been confirmed by negative time autocorrelation of toppling waves constituting an avalanche. A sandpile model having certain microscopic as well as macroscopic features of BTW follows FSS as that of MSM is a new result. The appearance of FSS in RSM may be due to local toppling imbalance and "internal stochasticity" caused by the imposed rotational constraint on the model.

Acknowledgment: The authors thank S. S. Manna, D. Dhar and M. A. Munoz for critical comments and several suggestions on the manuscript. This work is financially supported by Board of Research in Nuclear Sciences, Department of Atomic Energy, India, grant 
no.2005/37/5/BRNS.

[1] P. Bak, How Nature Works: the Science of Self-Organized Criticality, (Copernicus, New York, 1996); H. J. Jensen, Self-Organized Criticality (Cambridge, 1998),

[2] D. Dhar, Physica A 263, 4 (1999) and references therein.

[3] K. Christensen and N. R. Moloney, Complexity and Criticality (Imperial College Press, London, 2005).

[4] H. E. Stanley, Introduction to Phase Transitions and Critical Phenomena, (Oxford University Press, New York, 1971).

[5] For example: coagulation (H. Takayasu, Phys. Rev. Lett. 63, 2563 (1989)), earthquake (K. Chen, P. Bak and S. P. Obukhov, Phys. Rev. A 43, 625 (1990)), river network (H. Takayasu and H. Inaoka, Phys. Rev. Lett. 68, 966 (1992)), forest fire (B. Drossel, S. Clar and F. Schwabl, Phys. Rev. Lett. 71, 3739 (1993)), biological evolution (P. Bak and K. Snappen, Phys. Rev. Lett. 71, 4083 (1993)), dissolution of random solids (S. Sinha, V. Kishore and S. B. Santra, Europhys. Lett. 71, 632 (2005)), etc.

[6] P. Bak, C. Tang and K. Wiesenfeld, Phys. Rev. Lett. 59, 381 (1987); P. Bak, C. Tang and K. Wiesenfeld, Phys. Rev. A. 38, 364 (1988); C. Tang and P. Bak, Phys. Rev. Lett. 60, 2347 (1988).

[7] S. S. Manna, J.Phys.A:Math.Gen 24, L363(1991); Physica A 179, 249 (1991).

[8] L. Pietronero, A. Vespignani, and S. Zapperi, Phys. Rev. Lett, 72, 1690 (1994); A. Vespignani, S. Zapperi, and L. Pietronero, Phys. Rev. E, 51, 1711 (1995).

[9] A. Diaz-Guilera, Phys. Rev. A 45, 8551 (1992); Europhys. Lett. 26, 177 (1994).

[10] A. Chessa, H. E. Stanley, A. Vespignani, and S. Zapperi, Phys. Rev. E, 59, R12 (1999).

[11] K. Christensen and Z. Olami, Phys. Rev. E 48, 3361 (1993).

[12] A. Ben-Hur and O. Biham, Phys. Rev. E 53, R1317 (1996); E. Milshtein, O. Biham, and S. Solomon, Phys. Rev. E 58, 303 (1998).

[13] M. De Menech, A. L. Stella and C. Tebaldi, Phys. Rev. E 58, R2677 (1998); C. Tebaldi, M. De Menech and A. L. Stella, Phys. Rev. Lett. 83, 3952 (1999).

[14] S. Lübeck, Phys. Rev. E 61, 204 (2000).

[15] A. Chessa, A. Vespignanai, S. Zapperi, Comput. Phys. commun. 121, 299 (1999); A. Vespig- 
nanai, R. Dickman, M. A. Munoz, and S. Zapperi, Phys. Rev. E 58, R2677 (1998).

[16] B. K. Chakraborti and S. S. Manna, J. Phys. A 16, L113 (1983); S. Redner and I. Majid, J. Phys. A 16, L307 (1983).

[17] H. J. W. Blöte and H. J. Hilhorst, J. Phys. A 17, L111 (1984); K. Y. Lin, J. Phys. A 18, L145 (1985).

[18] T. C. Li and Z. C. Zhou, J. Phys. A18, 67 (1985); S. B. Santra and I. Bose J. Phys. A22, 5043 (1989).

[19] H. Hinrichsen, Adv. Phys. 49, 815 (2000) and references therein.

[20] S. B. Santra and I. Bose, J. Phys. A24, 2367 (1991).

[21] S. B. Santra, Eur. Phys. J. B 33, 75 (2003).

[22] D. Dhar and R. Ramaswamy, Phys. Rev. Lett. 63, 1659 (1989).

[23] R. Karmakar and S. S. Manna, Phys. Rev. E 71, R015101 (2005).

[24] R. Karmakar, S. S. Manna and A. L. Stella, Phys. Rev. Lett. 94, 088002 (2005).

[25] S. S. Manna and D. Giri, Phys. Rev. E 56, R4914 (1997).

[26] Y. C. Zhang, Phys. Rev. Lett. 63, 470 (1989).

[27] S. Lübeck, Phys. Rev. E 56, 1590 (1997).

[28] S. S. Manna, Current Science, 77, 388 (1999).

[29] O. Biham, E. Milshtein and O. Malcai, Phys. Rev. E 63, 061309 (2001).

[30] P. Grassberger and S. S. Manna, J. Phys (France) 51, 1077 (1990); S. S. Manna, J. Stat. Phys. 63, 653 (1991); S. Banerjee, S. B. Santra and I. Bose, Z. Phys. B 96, 571 (1995).

[31] S. S. Manna and A. L. Stella, Physica A 316, 135 (2002).

[32] S. Lübeck and K. D. Usadel, Phys. Rev. E 55, 4095 (1997).

[33] S. N. Majumder and D. Dhar, Physica A 185, 129 (1992).

[34] K. Christensen, H. C. Fogedby, and H. J. Jensen, J. Stat. Phys. 59, 509 (1990).

[35] R. Pastor-Satorras and A. Vespignani, J. Phys. A 33, L33 (2000); Phys. Rev. E. 62, 6195 (2000).

[36] S. B. Santra and W. A. Seitz, Int. J. Mod. Phys. C 11, 1357 (2000).

[37] V. B. Priezzhev, D. V. Ktitarev and E. V. Ivashkevich, Phys. Rev. Lett. 76, 2093 (1996); D. V. Ktitarev, S. Lübeck, P. Grassberger and V. B. Priezzhev, Phys. Rev. E 61, 81 (2000).

[38] M. De Menech and A. L. Stella, Phys. Rev. E 62, R4528 (2000); Physica A 309, 289 (2002); A. L. Stella and M. De Menech, Physica A 295, 101 (2001). 


\begin{tabular}{|c|c|c|c|}
\hline \multirow[b]{2}{*}{ Exponent } & \multicolumn{3}{|c|}{ Models } \\
\hline & BTW & MSM & RSM \\
\hline$\tau_{s}$ & 1.293 & 1.275 & $1.224 \pm 0.005$ \\
\hline$\tau_{a}$ & 1.330 & 1.373 & $1.334 \pm 0.005$ \\
\hline$\tau_{t}$ & 1.480 & 1.493 & $1.389 \pm 0.005$ \\
\hline$\tau_{l}$ & 1.665 & 1.743 & $1.667 \pm 0.007$ \\
\hline$\gamma_{s a}$ & 1.06 & 1.23 & $1.453 \pm 0.003$ \\
\hline$\gamma_{a t}$ & 1.53 & 1.35 & $1.167 \pm 0.005$ \\
\hline$\gamma_{a l}$ & 2.00 & 2.00 & $2.002 \pm 0.002$ \\
\hline$\gamma_{t l}$ & 1.32 & 1.49 & $1.715 \pm 0.005$ \\
\hline
\end{tabular}

TABLE I: Comparison of critical exponents obtained in different sandpile models. The values of the critical exponents for BTW and MSM are taken from Ref.[12, 32]. Some of the exponents are close to that of BTW and most of them are different from that of MSM.

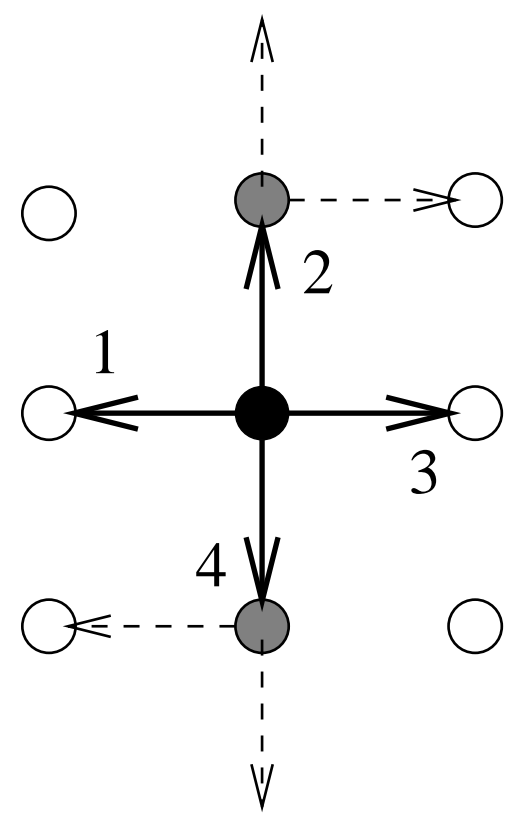

FIG. 1: RSM is demonstrated on a $3 \times 3$ square lattice. The central black site becomes upper critical first. Arrows with numbers 1-4 indicates four possible directions on the square lattice. Two sand grains flow along directions 2 and 4. Consequently, the grey sites become critical. The possible directions of flow of sands from the grey sites are indicated by dotted arrows. 


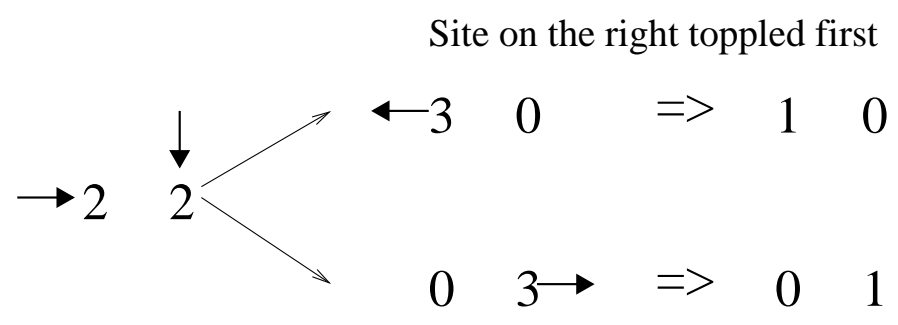

Site on the left toppled first

FIG. 2: Two different final states are obtained interchanging the toppling sequence starting from the same initial state. The numbers represent the height of the sandpile and the associated arrows represent the direction from which the last sand grain was received.

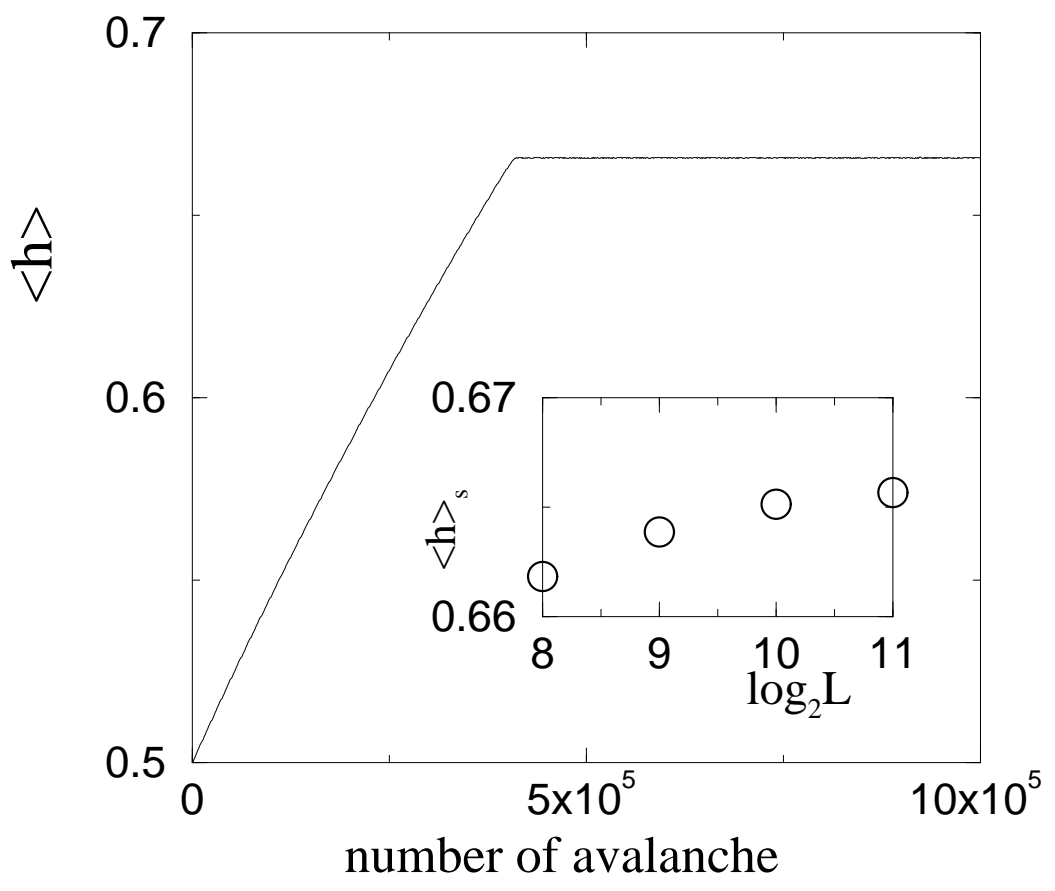

FIG. 3: Plot of average height $\langle h\rangle$ against the number of avalanches. The value of $\langle h\rangle$ remains constant over a large number of avalanches and changes slightly with the system size $L$. Dependence of the saturated average height $\langle h\rangle_{s}$ on the system size $L$ is shown in the inset. 


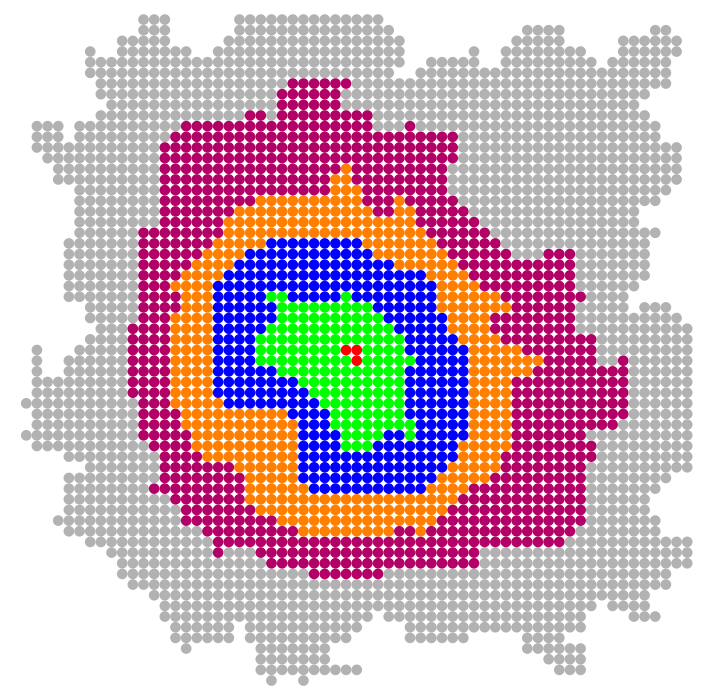

(a) BTW

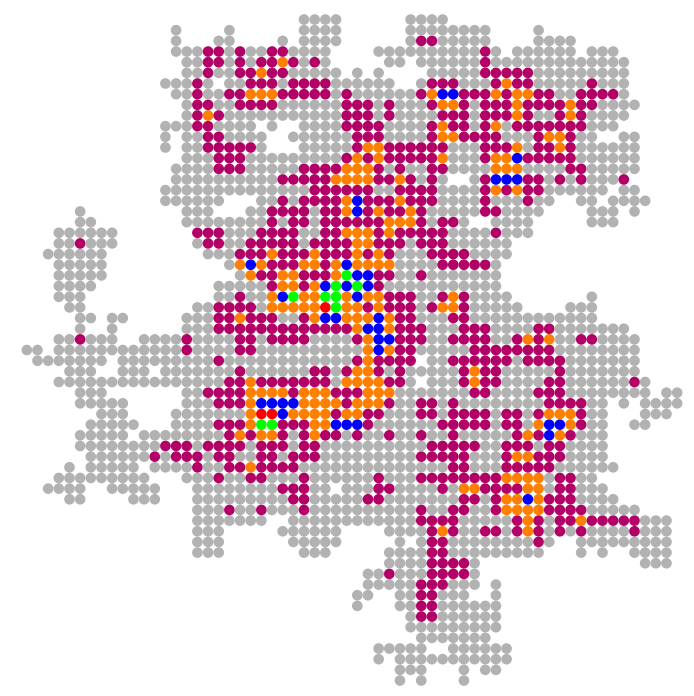

(b) MSM

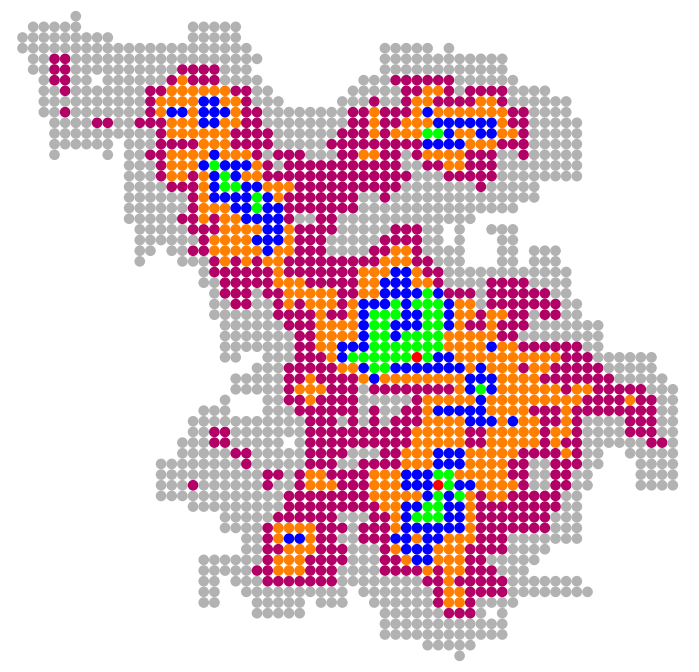

(c) RSM

FIG. 4: (Color online) Typical avalanches generated at the steady state are shown for BTW $(a)$, $\operatorname{MSM}(b)$, and RSM $(c)$ on a square lattice of size $64 \times 64$. Avalanches are generated dropping sand grains at the central site of the lattice. Maximum number of toppling occurs in each clusters is 21 . Different colors chosen are: red for 21, green for 20-17, blue for 16-13, orange for 12-9, magenta for 8-5, and grey for 4-1 toppling numbers. White space inside the avalanche corresponds to the sites that did not topple at all during the avalanche. Avalanche cluster of RSM has characteristics of both BTW and MSM. 


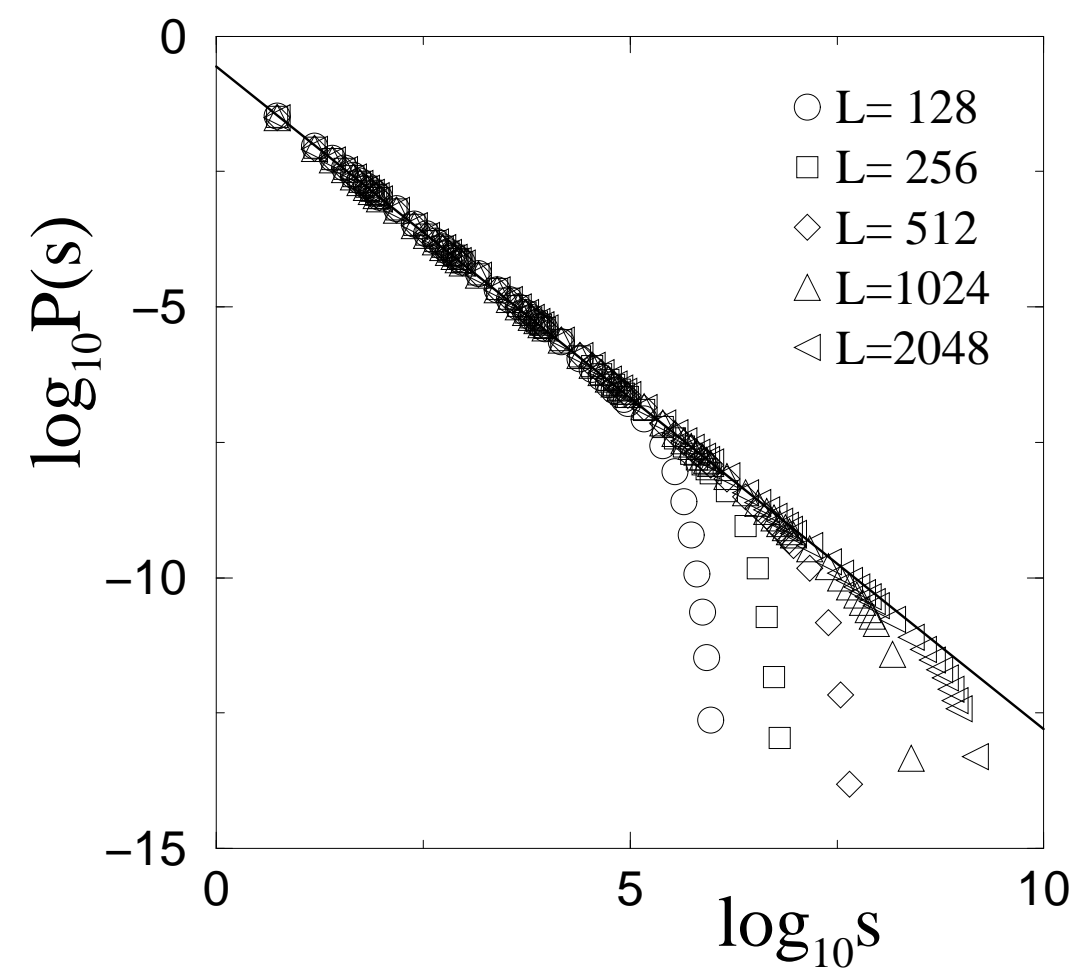

FIG. 5: $P(s)$ is plotted against $s$ for different system sizes $L: L=128(\bigcirc), L=256(\square), L=512$ $(\diamond), L=1024(\triangle), L=2048(\nabla)$. The solid lines show the best fitted part and the slope correspond to $\tau_{s}=1.224 \pm 0.005$. Toppling size distribution has power law correlation at the steady state. 


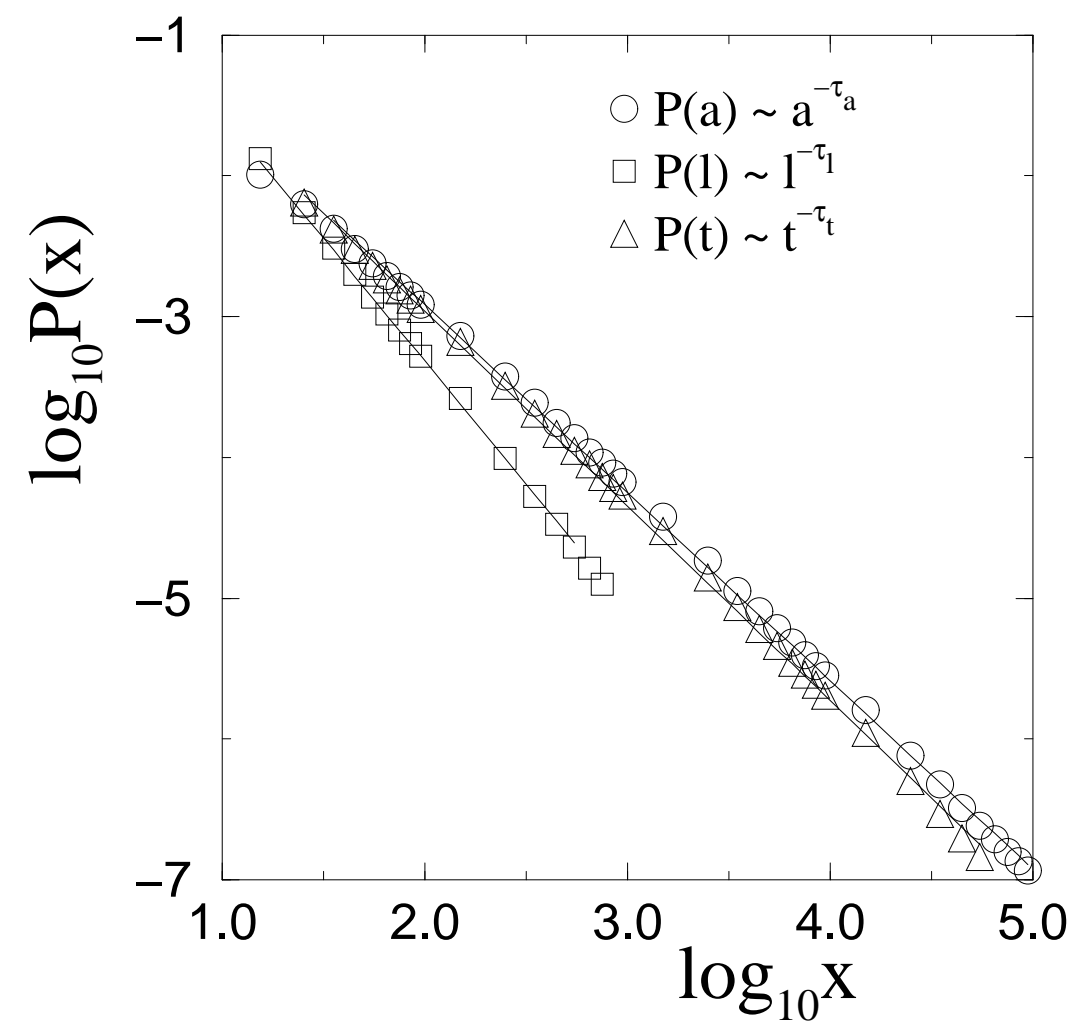

FIG. 6: Plot of probability distributions of avalanche area $P(a)(\bigcirc)$, lifetime $P(t)(\triangle)$ and extension of avalanche $P(l)(\square)$ against the corresponding variables $a, t$ and $l$ for $L=2048$. Reasonable power law distributions are obtained for all three properties. The solid lines show the best fitted parts and the slopes correspond to the respective exponents $\tau_{a}=1.334 \pm 0.005, \tau_{t}=1.389 \pm 0.005$ and $\tau_{l}=1.667 \pm 0.007$. Errors are least square fit error taking into account of statistical errors of each data points. 


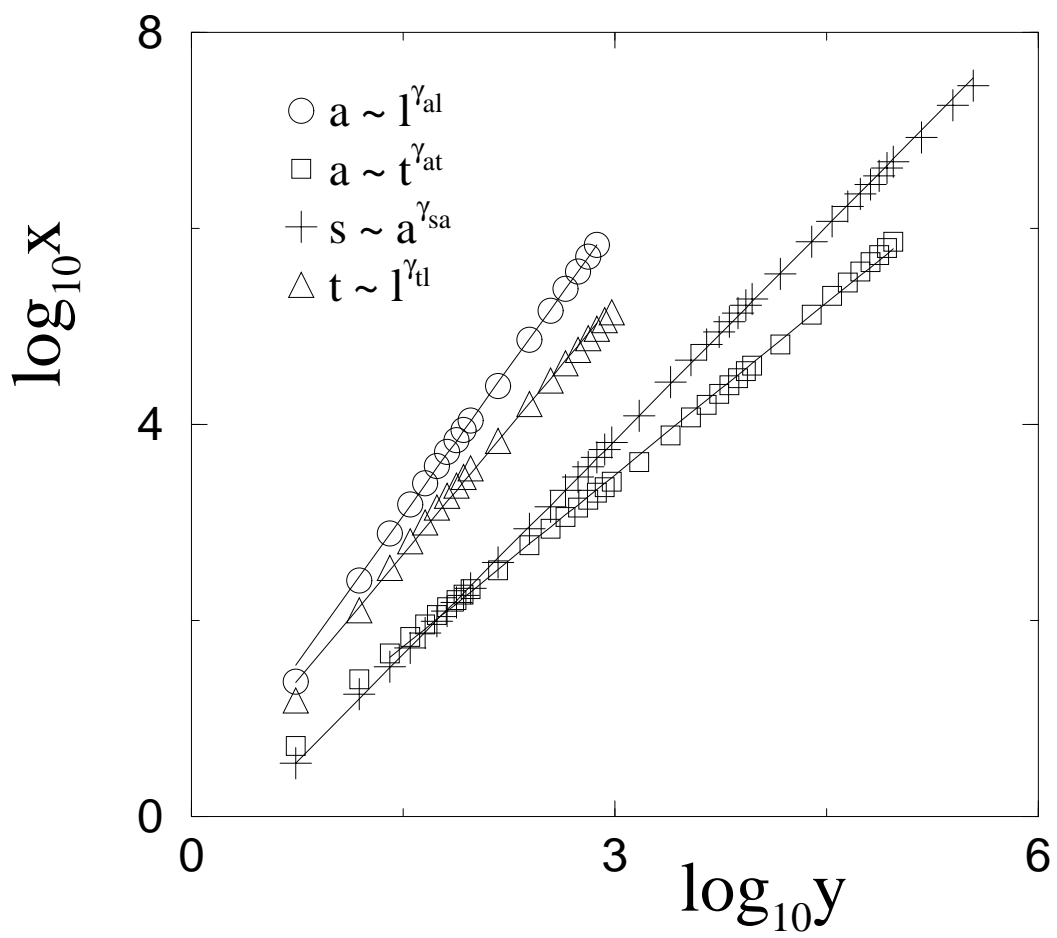

FIG. 7: Plot of conditional probabilities of avalanche properties: toppling $(s)$ versus area $(a)(+)$, area $(a)$ versus extension $(l)(\circ)$, area $(a)$ versus time $(t)(\square)$, and time $(t)$ versus length $(l)(\triangle)$. The solid lines show the best fitted straight line parts. Corresponding exponents are found as: $\gamma_{s a}=1.34 \pm 0.01, \gamma_{a t}=1.167 \pm 0.005, \gamma_{a l}=2.002 \pm 0.002$ and $\gamma_{t l}=1.713 \pm 0.015$. Errors are least square fit error. 


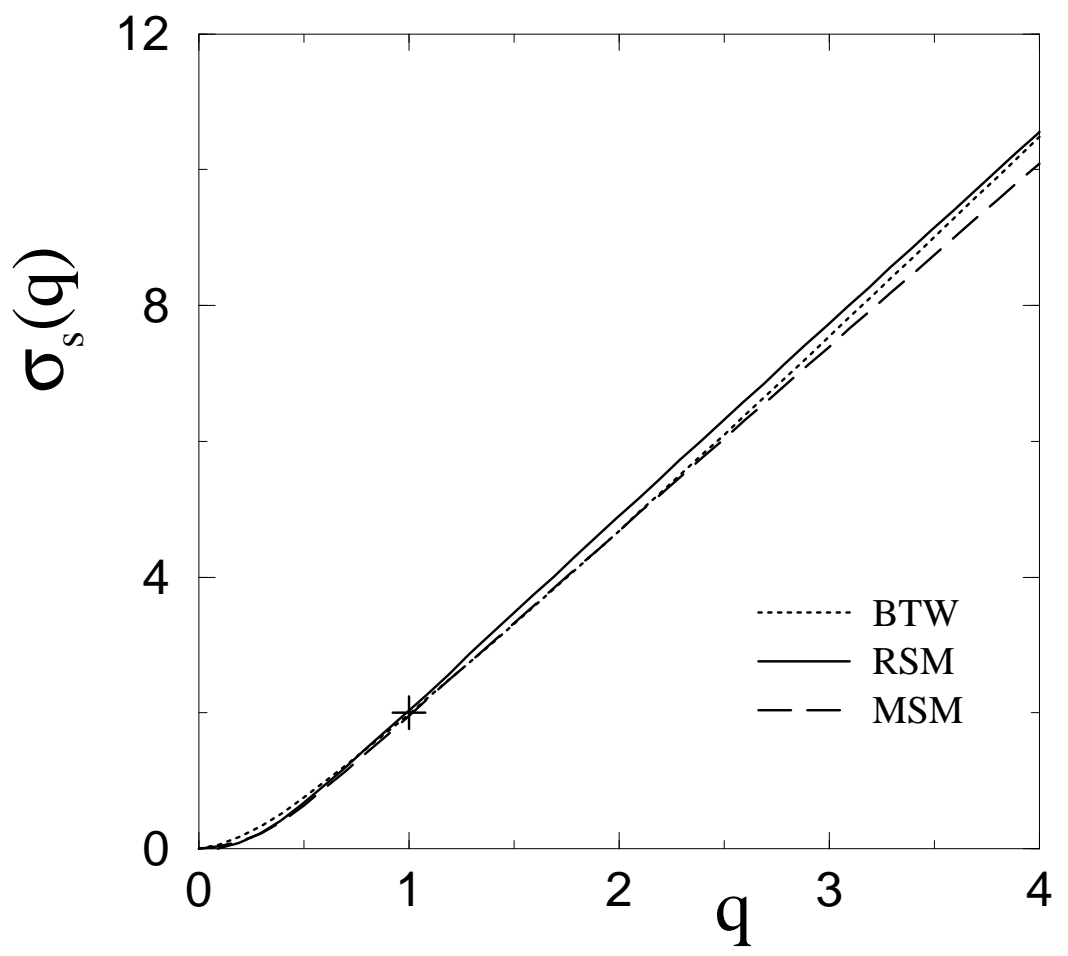

FIG. 8: Plot of $\sigma_{s}(q)$ versus $q$. Data of RSM (solid line) are compared with that of BTW (dotted line) and MSM (dashed line). $\sigma_{s}(q)$ of RSM is not identical with that of either BTW or MSM. Plus sign corresponds to the coordinate $q=1$ and $\sigma_{s}(1)=2$. 


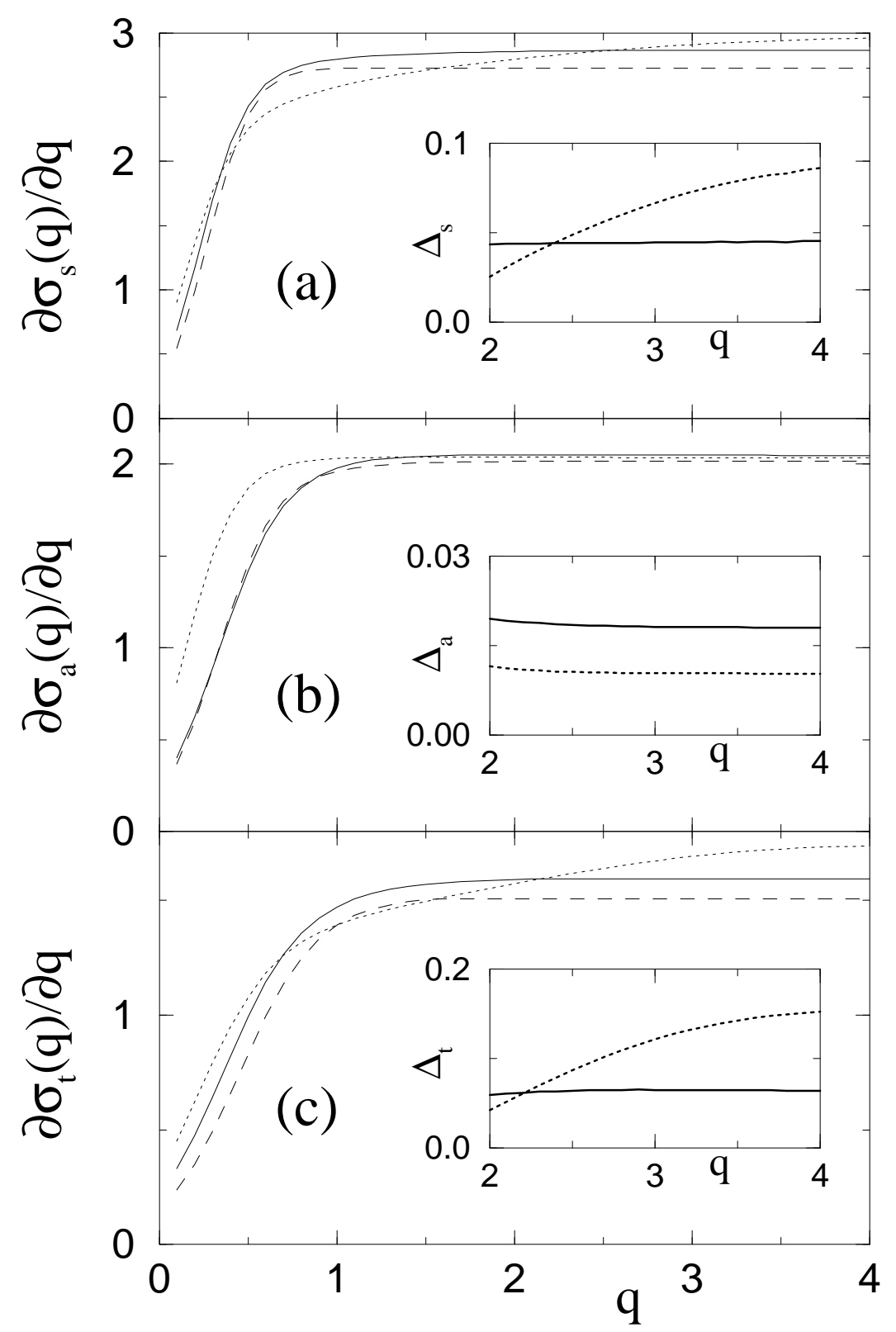

FIG. 9: Plot of $\partial \sigma_{x}(q) / \partial q$ versus $q$ where $x \in\{s, a, t\}$ in $(a),(b)$ and $(c)$ respectively. Data of RSM (solid line) are compared with that of BTW (dotted line) and MSM (dashed line). The rate of change of $\sigma_{x}$ s remain constant for RSM as in the case of MSM. In case of BTW, $\partial \sigma_{s}(q) / \partial q$ and $\partial \sigma_{t}(q) / \partial q$ do not saturate with the moment $q$. The relative increment of the rates $\Delta_{x}$ with respect to MSM are plotted against the moment $q$ in the inset of respective plots. Dotted lines represent the relative change of rates of BTW with respect to MSM and the solid lines represent the same for RSM with respect to MSM. 


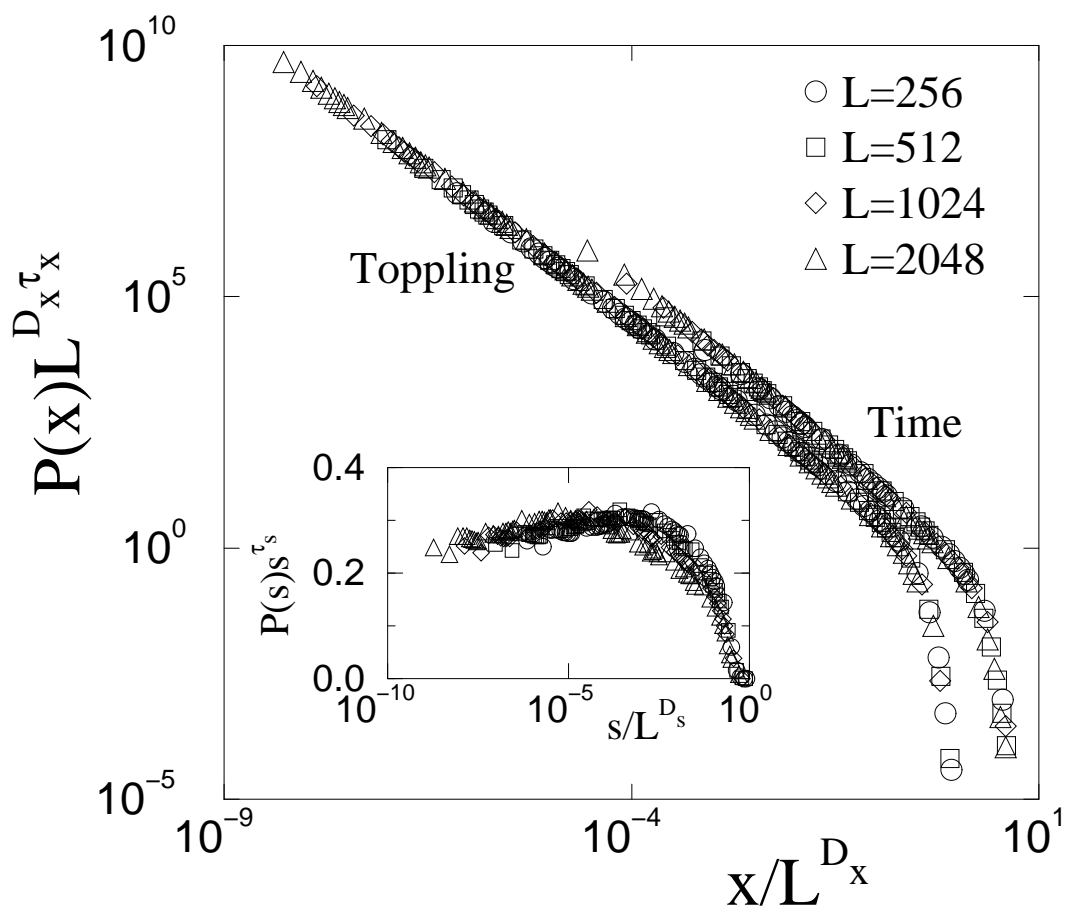

FIG. 10: Plot of the scaled distribution $P(x) L^{D_{x} \tau_{x}}$ against the scaled variable $x / L^{D_{x}}$ for $x \in\{s, t\}$. System sizes are taken as $L=256(\bigcirc), L=512(\square), 1024(\diamond)$ and $2048(\triangle)$. A reasonable data collapse occurs for both the toppling size $(s)$ and lifetime $(t)$ of the avalanche. In the inset, $P(x) s^{\tau_{x}}$ is plotted against $s / L^{D_{s}}$ is plotted in log-normal scale for the same system sizes. 


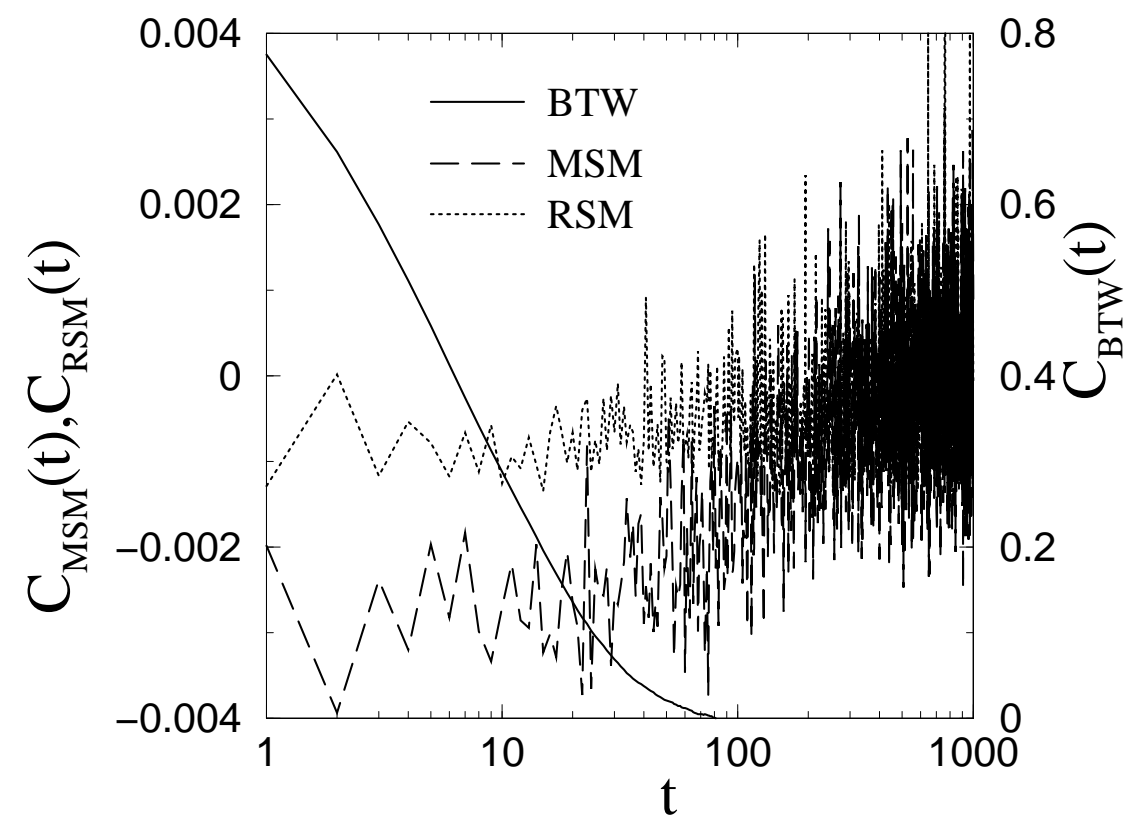

FIG. 11: Time auto correlation function $C(t)$ is plotted against time $t$ for BTW (solid line), MSM (dashed line) and RSM (dotted line). There is a long range correlation for BTW. The toppling waves are uncorrelated for both MSM and RSM. 